\title{
Editorial
}

\section{Mobile systems and applications}

\author{
Leonard Barolli ${ }^{\mathrm{a}}$, Hui-Huang $\mathrm{Hsu}^{\mathrm{b}}$ and Yoshitaka Shibata ${ }^{\mathrm{c}}$ \\ ${ }^{a}$ Department of Information and Communication Engineering, Faculty of Information Engineering, \\ Fukuoka Institute of technology (FIT), 3-30-1 Wajiro-Higashi, Higashi-ku, Fukuoka 811-0295, Japan \\ E-mail: barolli@fit.ac.jp \\ ${ }^{\mathrm{b}}$ Department of Computer Science and Information Engineering, Tamkang University, 151 Ying-Chuan \\ Road, Tamsui, Taipei, 25137, Taiwan \\ E-mail: hhsu@cs.tku.edu.tw \\ ${ }^{\mathrm{c}}$ Faculty of Software and Information Science, Iwate Prefectural University, 152-52 Sugo, Takizawa, \\ Takizawa-mura 020-0193, Iwate, Japan \\ E-mail: shibata@iwate-pu.ac.jp
}

In recent years, there has been rapid development in mobile communications and deployment of wireless communication infrastructure. For wireless communications, the dominant technologies have been the IEEE 802.11 WLAN and various cellular networks. The Bluetooth technology provides shortrange connectivity, and is poised to become a standard for personal area networking. On the device side, there is a wide spectrum of devices including laptops, palmtops, PDA and cell phones. Advances in these technologies have engendered a new paradigm of computing. Users now have the opportunity in accessing information anywhere and at any time. Ubiquitous computing is an emerging field of research for computing models in the 21 st century. This emergence is the natural result of research and technological advances mainly in wireless communications, mobile computing, embedded computing, autonomic computing, and agent technologies.

The aim of this special issue is to present the innovative researches, technologies as well as developments related to mobile networking, wireless communications and their applications. This special issue on Mobile Systems and Applications is organized with the papers presented at the IEEE 21st International Conference on Advanced Information Networking (AINA-2007) and fifteen workshops and symposiums in conjunction with AINA-2007, which were held at Niagara Falls, Ontario, Canada, May 21-23, 2007. The IEEE AINA-2007 conference received 444 paper submissions and every paper was reviewed carefully by at least three reviewers based on their quality, originality, and significance. Based on the review results, 134 papers were accepted for presentation. Also, from the workshops and symposiums, 334 papers were accepted. All the authors of AINA-2007 conference, workshops, and symposiums were encouraged to submit their revised manuscripts to this special issue. The submitted papers were reviewed carefully by at least three reviewers and based on the review results, 5 high-quality papers were accepted for publication in this special issue.

In the first paper, Guo et al. propose a new system named Home-Explorer that searches and finds physical artifacts in a smart indoor environment. The proposed system is an artifact-centered and uses 
sensors attached to the everyday artifacts in the real world. This paper makes two main contributions. First, it addresses, the robustness of the embedded sensors. The authors show that the current systems provide no mechanism to detect and manage hidden objects. Second, there is no common context infrastructure for building smart artifact systems, which makes it difficult for separately developed applications to interact with each other and uneasy for them to share and reuse knowledge. Different from previous systems, the proposed Home-Explorer uses an ontology-based knowledge infrastructure which makes it easy for the system to interact with other applications or agents also based on this ontology. The authors present and evaluate a set of rules for deducing an object's status or location information and for locating hidden objects.

Recent advances in the development of mobile terminals and the appropriate communication infrastructures have the consequence that new kinds of applications arise. This trend leads to more and more complex mobile client applications as well. In the second paper, Aleksy et al. introduce a generic architecture for the development of context-sensitive mobile applications. They also presented different techniques, which aim at conserving the resources and lowering the energy consumption of mobile terminals. The ability to download components when required conserves the limited storage of a mobile terminal and permits to adapt the application to the current user context. The proposed technique has advantages in case that the connection of the mobile application breaks down. If the just required component should already have been installed on the mobile terminal, then it can be employed by the user also in the case of an interruption of the communication. The proposed GUI framework overcomes the heterogeneity of the different Java libraries and takes into account the different technical conditions of mobile terminals. It also permits automatic adaptation of the user interface in case of context changes.

In the third paper, Durresi et al. deal with secure authentication in heterogeneous wireless networks. The convergence of cellular and IP technologies has pushed the integration of $3 \mathrm{G}$ and WLAN networks. Gaining secure access to $3 \mathrm{G}$ services from 802.11 WLANs is a primary challenge for this new integrated wireless technology. Successful execution of 3G security algorithms can be limited to a specified area by encrypting a user's authentication challenge with spatial data defining his visited WLAN. With limited capacity to determine a user's location only to within a current cell and restrictions on accessing users' location due to privacy, $3 \mathrm{G}$ operators must rely on spatial data sent from visited WLANs to implement spatial authentication control. A potential risk is presented to $3 \mathrm{G}$ operators since no prior relationship or trust may exist with a WLAN owner. The authors present some algorithms to quantify the trust between all parties of 3G-WLAN integrated networks to further secure user authentication. Ad-hoc serving networks and the trust relationships established between mobile users are explored to define stronger algorithms for 3G-WLAN user authentication. The authors show that with repeated proper performance and successful authentication requests, a serving network can be deemed a trusted partner in a hybrid 3G-WLAN network.

In the fourth paper, Aversa et al. present a skeleton based programming paradigm for mobile multiagents on distributed systems and its realization within the MAGDA mobile agent platform. They describe the mobile agent programming model and developed a framework that provides the programmer with high level programming skeletons. Skeletons implement automated mechanisms for agents' interaction exploiting migration and cloning. Algorithmic skeletons are presented as a specialization of the agent lifeline using some facilities provided by the exploited programming model. They allow to reuse parts of the sequential code by filling some methods, classes and interfaces, and to hide the difficulties to be faced with using an explicit parallel programming paradigm. The authors evaluate by experiments execution of the mobile agent based branch and bound optimization application. They define a normalized speedup in order to filter the branching effect in experimental results. The authors show that the normalized speedup scales well with the number of processors for all the considered problem sizes. 
In the last paper, Ozaki et al. propose a fully redundant model for Sensor, Actuator and Device Network (SADN), where the sensor, actuator, and device functions are replicated in multiple nodes and each of sensor-actuator and actuator-device communication is realized in many-to-many type of communication protocols. In the proposed model, even if some nodes are faulty, the other nodes can perform requested tasks. Each sensor node is able to e send the sensed values to multiple actuator nodes and each actuator node can receive the sensed values from multiple sensor nodes, while multiple actuator nodes communicate with multiple replica nodes of a device. Even if some messages are lost and some nodes are faulty, device nodes can surely receive action requests required for sensed values and the actions can be performed. In this paper, they discuss a Semi-Passive Coordination (SPC) protocol for multiple actuator nodes and multiple sensor nodes. They also present an active coordination protocol for multiple actuator nodes and multiple actuation device nodes. The authors evaluate the proposed SPC protocol for the sensor-actuator coordination in terms of the number of messages exchanged among actuators and show that proposed protocol has a good performance.

As we conclude this overview, we would like to thank all the authors for submitting their papers, and the reviewers for sending the review results on time. In particular, we would like to address our special thanks to the Editor-in-Chief of IJMIS Dr. David Taniar, for his strong encouragement and support to engage in this endevour. 

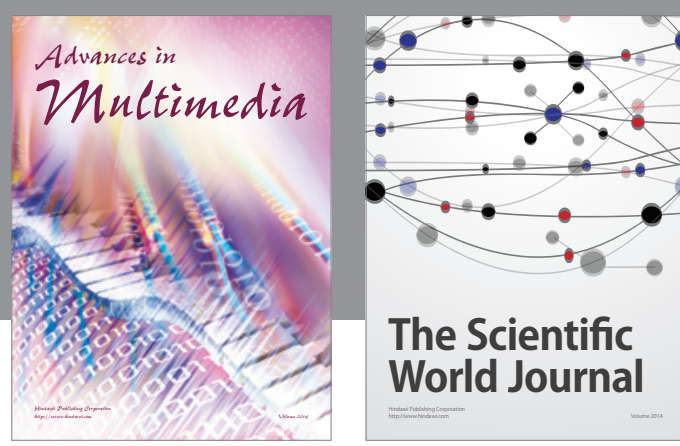

The Scientific World Journal
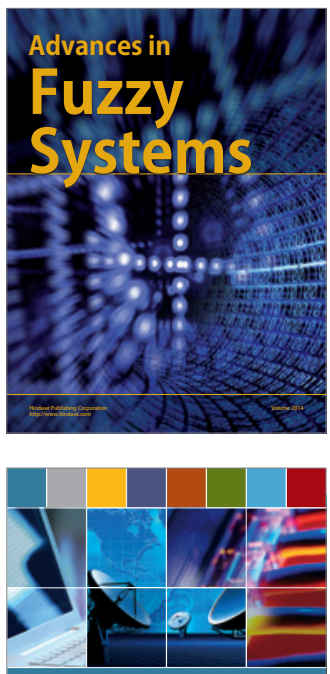

Computer Networks and Communications
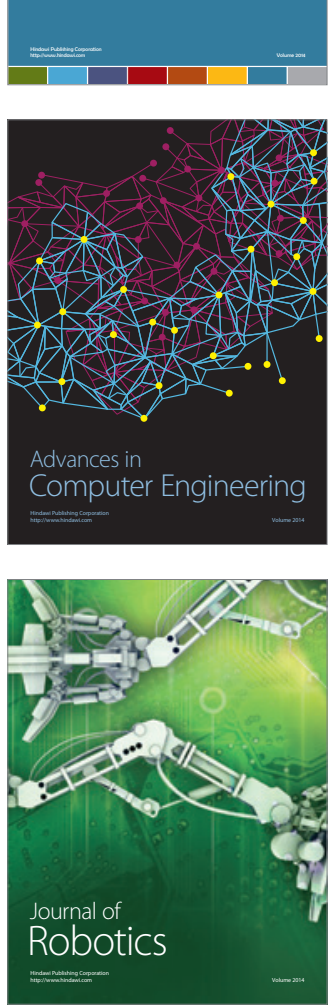
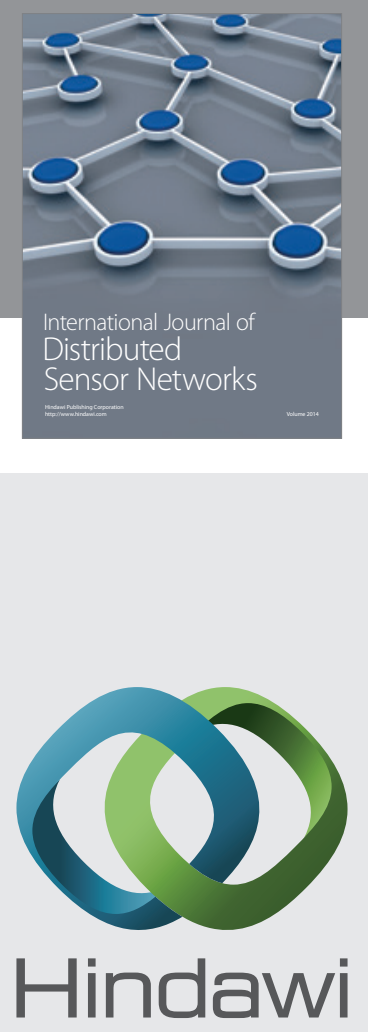

Submit your manuscripts at

http://www.hindawi.com
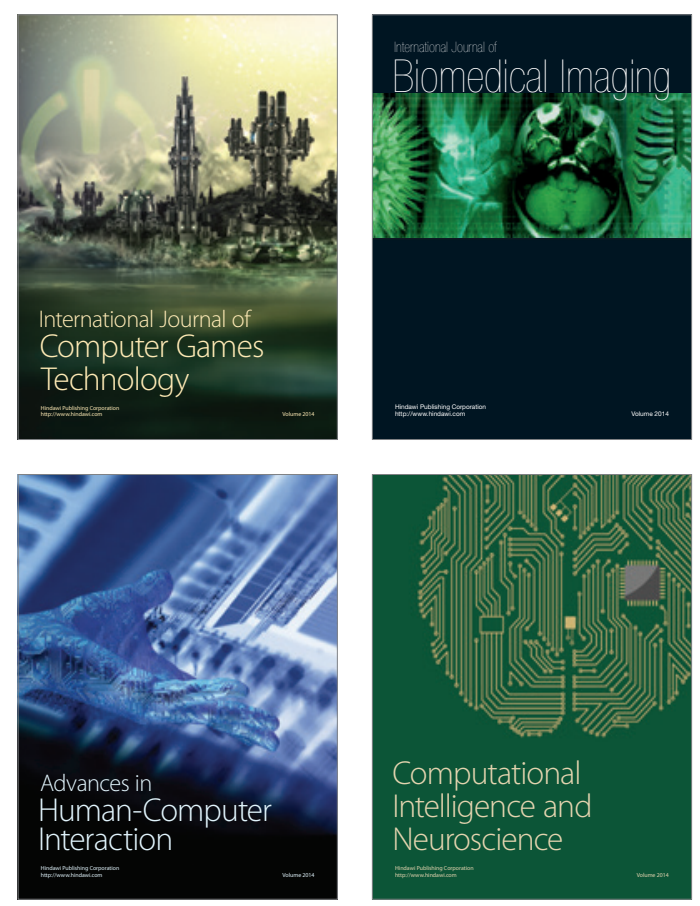
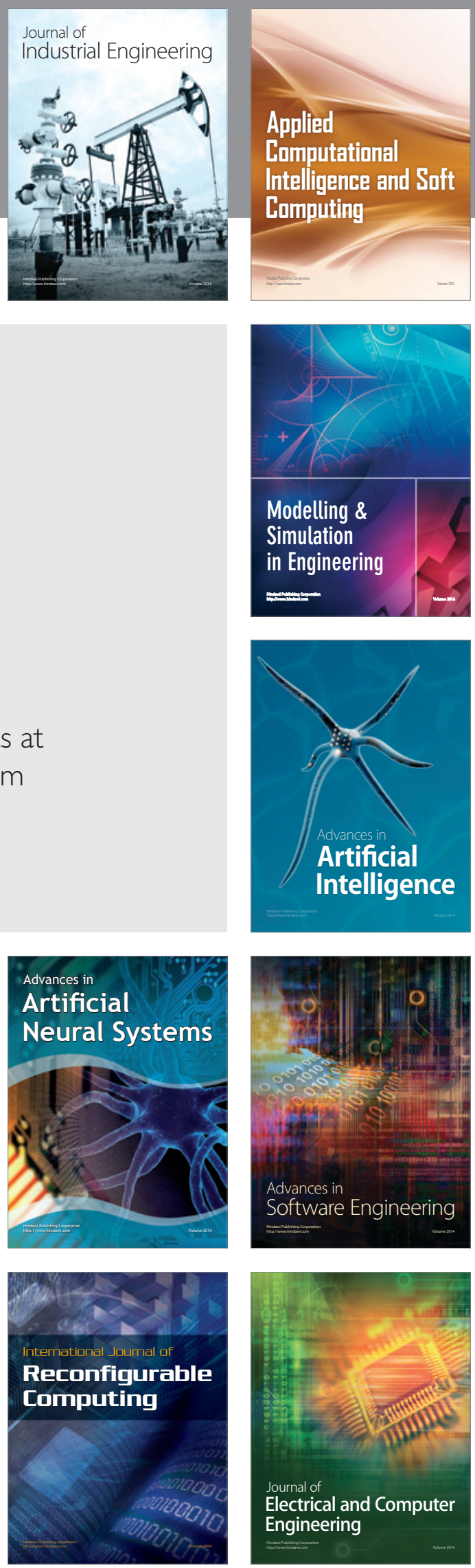